
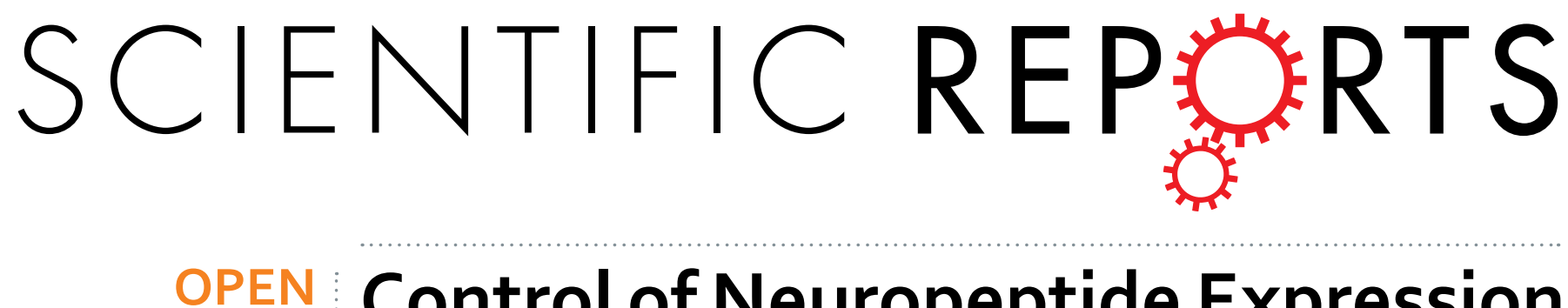

\title{
Control of Neuropeptide Expression by Parallel Activity-dependent Pathways in Caenorhabditis elegans
}

Received: 16 September 2016

Accepted: 14 November 2016

Published: 31 January 2017

\section{Teresa Rojo Romanos ${ }^{1,2}$, Jakob Gramstrup Petersen ${ }^{2}$ \&oger Pocock ${ }^{1,2}$}

Monitoring of neuronal activity within circuits facilitates integrated responses and rapid changes in behavior. We have identified a system in Caenorhabditis elegans where neuropeptide expression is dependent on the ability of the BAG neurons to sense carbon dioxide. In C. elegans, $\mathrm{CO}_{2}$ sensing is predominantly coordinated by the BAG-expressed receptor-type guanylate cyclase GCY-9. GCY-9 binding to $\mathrm{CO}_{2}$ causes accumulation of cyclic GMP and opening of the CGMP-gated TAX-2/TAX-4 cation channels; provoking an integrated downstream cascade that enables $C$. elegans to avoid high $\mathrm{CO}_{2}$. Here we show that $\mathrm{CGMP}$ regulation by GCY-9 and the PDE-1 phosphodiesterase controls BAG expression of a FMRFamide-related neuropeptide FLP-19 reporter (flp-19::GFP). This regulation is specific for $\mathrm{CO}_{2}$-sensing function of the BAG neurons, as loss of oxygen sensing function does not affect flp-19::GFP expression. We also found that expression of $f l p-19:: G F P$ is controlled in parallel to GCY-9 by the activity-dependent transcription factor CREB (CRH-1) and the CAMP-dependent protein kinase (KIN-2) signaling pathway. We therefore show that two parallel pathways regulate neuropeptide gene expression in the BAG sensory neurons: the ability to sense changes in carbon dioxide and CREB transcription factor. Such regulation may be required in particular environmental conditions to enable sophisticated behavioral decisions to be performed.

C. elegans performs multiple sensory modalities when navigating its environment. Such behaviors include sensing and responding to changes in temperature, atmospheric gases, food and mating ${ }^{1-8}$. Coordination of these complex behavioral responses requires constant monitoring of the status of the nervous system. Neuropeptide and neuropeptide receptor expression levels have previously been linked to the activity of neurons ${ }^{4,9-13}$. For example, insulin and neuropeptide signaling have been shown to report feeding status ${ }^{14}$. More recently, the regulation of expression of the serpentine receptor srh-234 was linked with starvation, and dependent on the neuropeptide $\mathrm{Y}$ receptor NPR-1 and insulin-like growth factor receptor DAF-2 ${ }^{12}$.

Despite having a small nervous system, the C. elegans genome encodes many neuropeptides ${ }^{15}$. These neuropeptides are classified into three main families: insulin-like family, neuropeptide-like protein family (NLPs) and FMRFamide-related peptides (FLPs). For FLP neuropeptides, the function for only a few are known suggesting that they work redundantly or are involved in fine-tuning of the nervous system in combination with neurotransmitter systems. However, certain functions have been elucidated for FLPs: FLP-11 has been identified as a regulator of a sleep-like state ${ }^{16}$, FLP-13 acts in the ALA neurons to regulate quiescence after heat-stress ${ }^{17}$, FLP-10 and FLP-17 inhibit egg laying ${ }^{18}$ and FLP-21 regulates social feeding behavior through the NPR-1 receptor ${ }^{19}$.

The C. elegans nervous system consists of 302 neurons, some of which function in non-overlapping circuits to regulate distinct behaviors. The BAG neurons for example are major regulators of acute $\mathrm{CO}_{2}$ avoidance behavior ${ }^{5,6}$, the regulation of pharyngeal pumping rate when animals are exposed to high levels of $\mathrm{CO}_{2}{ }^{20}$, and the control of egg laying ${ }^{18}$. The BAG neurons also act in conjunction with the URX neurons to coordinate behavioral responses to changes in $\mathrm{O}_{2}$ gradients. The BAG and URX neurons are activated when $\mathrm{O}_{2}$ levels drop and increase respectively $y^{3,21-23}$. However, the relationship between the BAG and URX neurons is not exclusive to $\mathrm{O}_{2}$ sensing: BAG-ablated animals live longer, while ablation of the URX neurons results in shorter lifespan, and these physiological changes are regulated by $\mathrm{O}_{2}$-sensing guanylate cyclases expressed in these neurons ${ }^{24}$. With such divergent

${ }^{1}$ Development and Stem Cells Program, Monash Biomedicine Discovery Institute and Department of Anatomy and Developmental Biology, Monash University, Melbourne, Victoria 3800, Australia. ${ }^{2}$ Biotech Research and Innovation Centre, University of Copenhagen, Ole Maaløes Vej 5, Copenhagen, Denmark. Correspondence and requests for materials should be addressed to R.P. (email: roger.pocock@monash.edu) 
and sophisticated roles for the BAG and URX neurons, prioritization and integration of information to guide behavioral responses may require modular regulation of neuropeptide expression.

Here we describe a system where the activity of the BAG neurons is crucial for the expression of a transcriptional reporter for the FLP-19 neuropeptide $(f l p-19:: G F P)$. Furthermore, we show that the expression of flp-19::GFP is regulated via two parallel modules: 1) cGMP signaling through GCY-9 and PDE-1 and 2) the CREB transcription factor.

\section{Results}

flp-19::GFP Expression in the BAG Neurons Requires Cilia Function. It has previously been shown that neuropeptide expression is highly plastic, and can be controlled by neuronal activity ${ }^{4,10,13}$. The $f l p-19:: G F P$ transcriptional reporter drives expression in the BAG, URX, AIN, AWA and HSN neurons in the hermaphrodite ${ }^{25}$. Our previous studies showed that $f l p-19:: G F P$ expression in the BAG neurons is exquisitely sensitive to perturbations in parallel transcriptional pathways that control BAG cell fate and function ${ }^{26-28}$. We hypothesized that neuronal activity may underpin the transcriptional regulation of $f l p-19:: G F P$ in the BAG neurons. We therefore tested if the expression of $f l p-19:: G F P$ is compromised when the activity of the BAG neurons is reduced or abolished.

As the BAG neurons are ciliated, we first examined if disruption of cilia structure, which is required for several behaviors in C. elegans $s^{7,29,30}$, would affect $f l p-19:: G F P$ expression. We crossed the $f l p-19:: G F P$ reporter (ynIs34) with a mutant of che-3, which encodes a dynein that is required for structural integrity of sensory cilia. We observed that che-3(e1379) mutant animals exhibit strong defects in the expression of $f l p-19:: G F P$ in the ciliated BAG and AWA neurons but not in the non-ciliated neurons (Fig. 1A,B). To verify this regulation, we crossed che-3(e1379) mutant animals with an independent $f l p-19:: G F P$ reporter ( $r p E x 811)$ and observed a similar phenotype (Fig. 1C). We next asked if disturbance of cilia transport would produce a similar effect on flp-19::GFP expression. TUB-1, homolog of TUBBY in mammals, is required for correct localization of $\mathrm{G}$ protein coupled receptors to cilia ${ }^{31-33}$. We found that removal of TUB-1 function phenocopied the che-3 mutant loss of $f l p-19:: G F P$ expression in the BAG neurons (Fig. 1A,B), indicating that correct cilia function is required for expression of $f l p-19:: G F P$.

GCY-9 Regulates flp-19::GFP Expression Cell-autonomously in the BAG Neurons. Acute responses to $\mathrm{CO}_{2}$ are regulated by a neuronal circuit that includes the BAG neurons. The BAG neurons sense carbon dioxide through the GCY-9 receptor-type guanylate cyclase, homolog of the human GC-D $26,34,35$. As such, $g c y-9$ mutants are unable to sense and respond to changes in $\mathrm{CO}_{2}$ concentration ${ }^{34}$. We asked whether the $\mathrm{CO}_{2}$-sensing function of the BAG neurons is required for $f l p-19:: G F P$ expression. To this end, we crossed two independent deletion alleles of $g c y-9(n 4470, t m 2816)$ with the $f l p-19:: G F P$ reporter and observed a reduced number of animals expressing GFP in the BAG neurons (Fig. 2A,B). When we resupplied $g c y-9 c D N A$ driven by a BAG specific $g c y$-33 promoter into $g c y-9(n 4470)$ mutant animals, $f l p-19:: G F P$ expression was restored (Fig. 2B). The BAG neurons are also involved in sensing downshifts in $\mathrm{O}_{2}$ concentration, through expression of the soluble guanylate cyclases GCY-31 and GCY-33 ${ }^{22}$. To ask whether $\mathrm{O}_{2}$-sensing function of the BAG neurons is also required to regulate $f l p-19:: G F P$ expression, we crossed $g c y$-31(ok296) and $g c y$-33(ok232) mutants with the flp-19::GFP reporter. We found no detectable change in expression of $f l p-19:: G F P$ when $\mathrm{BAG} \mathrm{O}_{2}$-sensing function was ablated (Fig. 2C). As it has been previously shown that the URX and BAG communicate with each other ${ }^{23,24}$, we asked whether removal of $\mathrm{O}_{2}$-sensing from the URX neurons would affect the expression of $f$ lp-19::GFP. We therefore crossed the flp-19::GFP reporter into $g c y$-35(ok769) and $g c y$-36(db42) mutant strains, in which soluble guanylate cyclases required for $\mathrm{URX}_{2}$-sensing function are mutated ${ }^{3,4,22,36}$. However, we observed no defect in the expression of $f l p-19:: G F P$ in the BAG or URX neurons (Fig. 2C and data not shown). Furthermore, animals which lack $\mathrm{O}_{2}$ sensing function in both the BAG and URX neurons ( $g c y$-31 gcy-36; gcy-33; gcy-35 quadruple mutant) exhibit wild type expression of $f l p-19:: G F P$ (Fig. 2C). Taken together, these data show that $\mathrm{CO}_{2}$ sensing function, and not $\mathrm{O}_{2}$ sensing function, regulates the expression of the $f l p-19:: G F P$ reporter in the BAG neurons.

To ask whether $\mathrm{CO}_{2}$ sensing has a general effect on neuropeptide expression in the BAG neurons, we analyzed the expression of other neuropeptides in $g c y$-9(n4470) mutant animals. We crossed the $g c y$ - $9(n 4470)$ mutant with reporters for $f l p-17(y n I s 64)$ and $f l p-13(y n I s 37)$ and observed no change of expression compared to wild type (Figure S1A). Furthermore we tested other reporters expressed in the BAG neurons (soluble guanylate cyclases $g c y$-31(rpIs29) and $g c y$-33(rpIs7) and the transcription factor egl-13(rpIs32)) in the $g c y-9$ mutant background and we observed no change of expression (Figure S1A). Therefore, the regulation of $f l p-19:: G F P$ expression by GCY-9 is somewhat specific.

cGMP Levels Regulate flp-19::GFP Expression in the BAG Neurons. $g c y$-9 encodes a receptor-type guanylate cyclase. The role of these enzymes is to generate cGMP for gating downstream cyclic nucleotide-gated TAX-2/TAX-4 cation channels. As such GCY-9 regulates the activity of the BAG neurons through the control of cGMP levels. We speculated therefore that cGMP is a key regulator of $f l p-19:: G F P$ expression in the BAG neurons. In order to test this hypothesis, we genetically manipulated cGMP levels. Phosphodiesterases are enzymes that catalyse the breakdown of cGMP to GMP and it has previously been shown that the phosphodiesterase PDE-1 is expressed in the BAG neurons ${ }^{34}$. We therefore asked whether the predicted increase in cGMP levels in $p d e-1$ mutant animals would affect $f l p-19:: G F P$ expression. We crossed the $p d e-1$ (nj57) mutant with the $f l p-19:: G F P$ reporter and observed that BAG expression of $f l p-19:: G F P$ was unaffected (Fig. 3A). Next we tested if loss of $p d e-1$, and therefore increase of cGMP in the BAG neurons, was sufficient to derepress $f l p-19:: G F P$ expression in the $g c y$-9 mutant. We therefore examined flp-19::GFP expression in the pde-1(nj57); $g c y-9(n 4470)$ double mutant and found that expression in the BAG neurons was fully restored (Fig. 3A). This suggests that reduced levels of cGMP in the BAG neurons causes transcriptional downregulation of $f l p-19:: G F P$. 
A

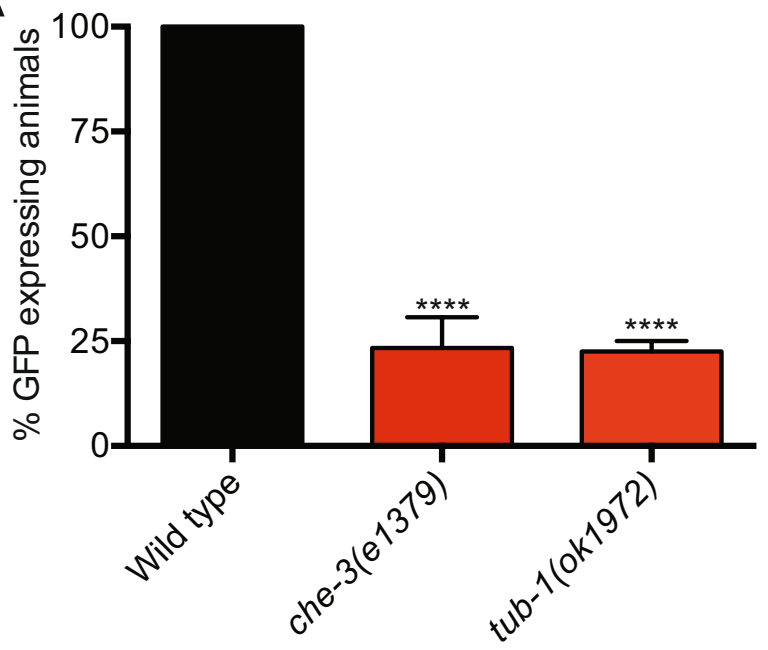

B
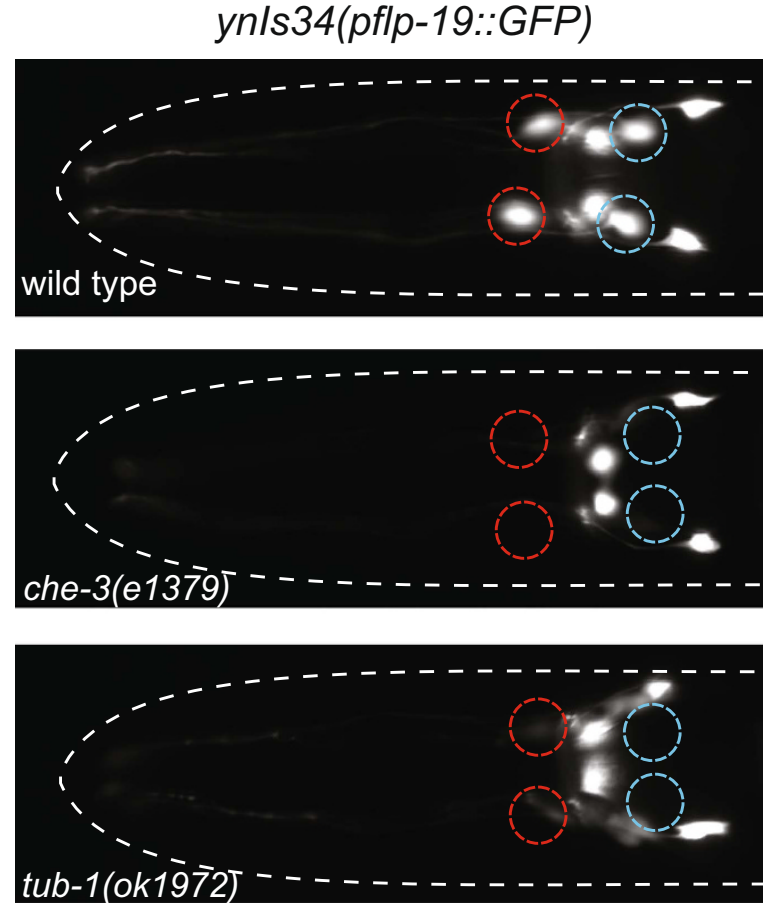

C

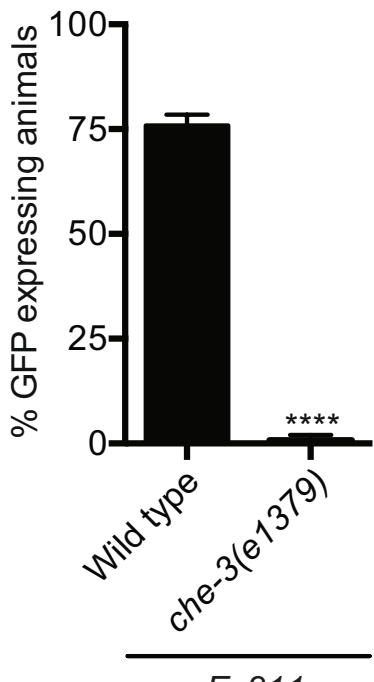

$r p E x 811$

Figure 1. Cilia Function is Required for Expression of flp-19::GFP in the BAG Gas-sensing Neurons. (A) Quantification of the expression of the $f l p-19:: G F P$ fluorescent reporter ( $y n I s 34)$ in wild type, che-3(e1379) and $t u b-1$ (ok1972) mutant animals. $\mathrm{n}>50$. ${ }^{* * *} \mathrm{P}<0.0001$. See Materials and Methods for neuronal scoring criteria used. (B) Micrographs show representative pictures of $f l p-19:: G F P(y n I s 34)$ expression in wild type, che-3(e1379) and tub-1(ok1972) strains. BAG positions are marked with red dashed circles. Note - expression of $f l p-19:: G F P$ is also lost in the ciliated AWA neurons in che-3(e1379) and tub-1(ok1972) mutant animals (blue dotted circles). Anterior to the left. Scale bar $=20 \mu \mathrm{m}$. (C) Quantification of the expression of the flp-19::GFP reporter $(r p E x 811)$ in wild type and che-3(e1379) backgrounds. These data confirm the results shown in Fig. 1A. $\mathrm{n}>50 .{ }^{* * *} \mathrm{P}<0.0001$.

Activation of GCY-9 by $\mathrm{CO}_{2}$ normally triggers the conversion of cGMP from GTP ${ }^{35}$. Subsequently, cGMP opens the cyclic nucleotide-gated channels TAX-2/TAX-4, through which the neuron is activated by calcium influx ${ }^{5,6}$. We therefore hypothesized that GCY-9-mediated regulation of the $f l p-19:: G F P$ reporter was through this canonical pathway. Indeed, we found that in tax-4(p678) mutant animals $f l p-19:: G F P$ expression is undetectable in the BAG neurons (Fig. 3B). In addition, we observed loss of $f l p-19:: G F P$ expression in the $\mathrm{URX}_{2}$-sensing neurons of tax-4(p678) mutant animals, suggesting that similar mechanisms of regulation may exist in these 
A

gcy-9(n4470); flp-19::GFP

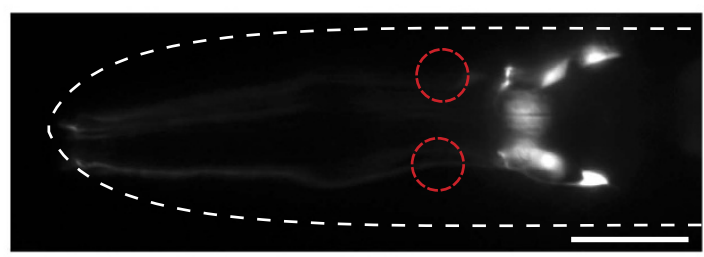

gcy-9(tm2816); flp-19::GFP

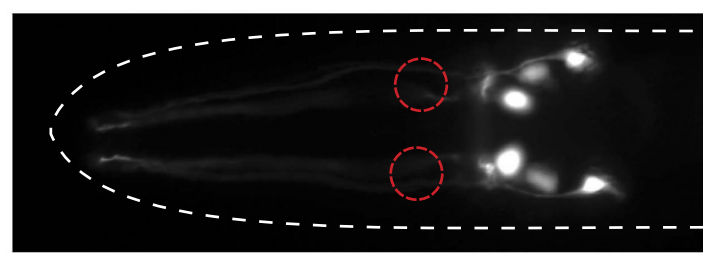

B

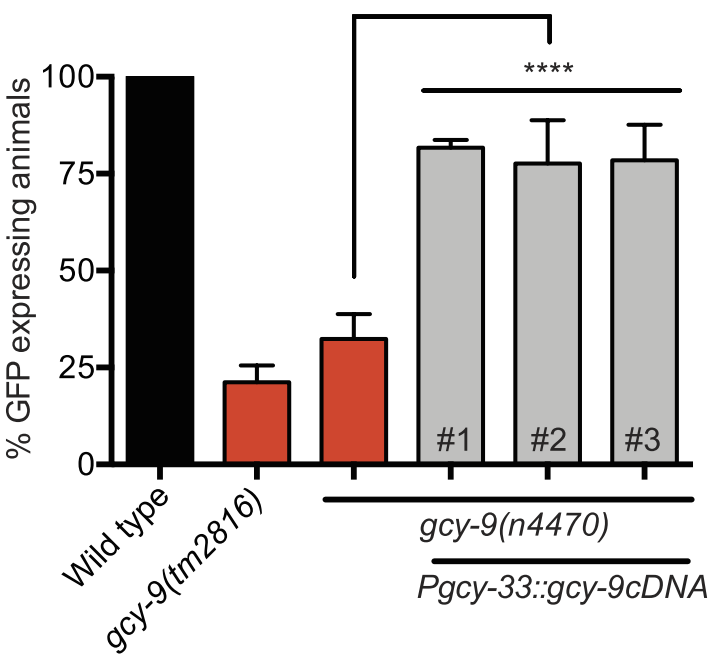

C

\begin{tabular}{|c|c|c|}
\hline Strain & defects in BAGs & $\mathbf{n}$ \\
\hline gcy-31(ok296) & NO & 52 \\
\hline$g c y-33(o k 232)$ & NO & 51 \\
\hline$g c y-35(o k 769)$ & NO & 39 \\
\hline$g c y-36(d b 42)$ & NO & 49 \\
\hline$g c y-33 ; g c y-35 ; g c y-36$ gcy-31 & NO & 90 \\
\hline
\end{tabular}

Figure 2. Autonomous $\mathrm{CO}_{2}$ Sensing is Required for $f l p-19$ :: GFP Expression in the BAG Neurons.

(A) $g c y-9(n 4470)$ and $g c y-9(t m 2816)$ mutants exhibit reduced expression of the $f l p-19:: G F P$ reporter in the BAG neurons. Micrographs show representative images of $g c y-9$ mutant hermaphrodites expressing the $f l p-19:: G F P$ reporter (compare to Fig. 1B, top panel). BAG positions are marked with red dashed circles. Anterior to the left. Scale bar $=20 \mu \mathrm{m}$. (B) Quantification of $f l p-19:: G F P$ expression in $g c y-9(n 4470)$ and $g c y-9(t m 2816)$ mutant animals. Transgenic expression of $g c y$ - $9 c D N A$ under the control of a BAG-specific $g c y$-33 promoter rescues $g c y-9(n 4470)$ mutant phenotype. \#1-3 indicate independent transgenic rescue lines. See Materials and Methods for neuronal scoring criteria used. $\mathrm{n}>50$. ${ }^{\star * \star} \mathrm{P}<0.0001$. (C) Table showing the effect of loss of $\mathrm{O}_{2}$-sensing guanylate cyclases on $f l p-19:: G F P$ expression in the BAG neurons. BAG guanylate cyclase functional nulls $g c y-$ 31 (ok296) and $g c y$-33(ok232) do not exhibit defects in expression of $f l p-19:: G F P$ in the BAG neurons. Mutants for URX guanylate cyclases $g c y$-35(ok769) and $g c y$-36(db42) do not exhibit defects in expression of $f l p-19:: G F P$ in the BAG neurons. The quadruple $g c y$-31 $g c y$-36; $g c y$-33; $g c y$-35 also does not exhibit any defects in BAG expression suggesting that $f l p-19:: G F P$ expression in the BAG neurons is dependent on $\mathrm{CO}_{2}$ sensing and not $\mathrm{O}_{2}$ sensing. 


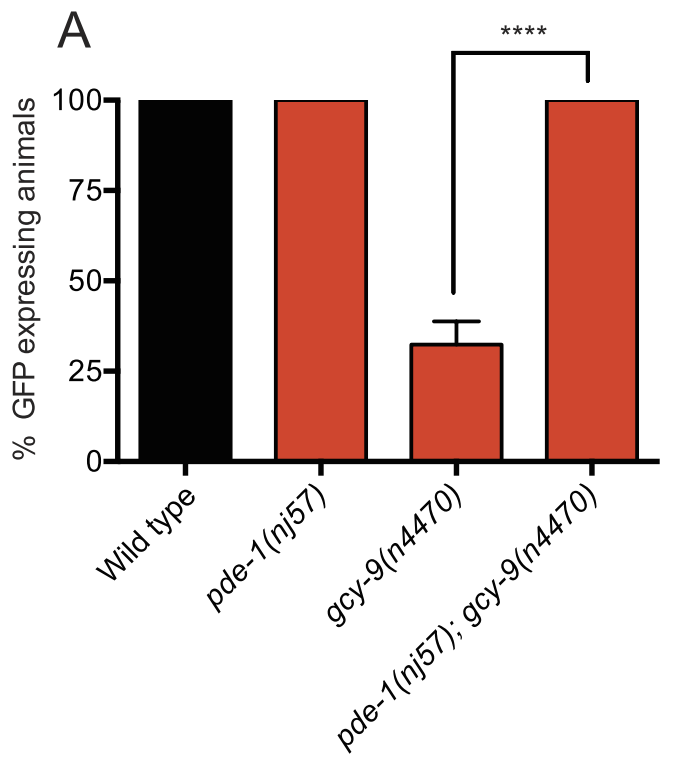

B
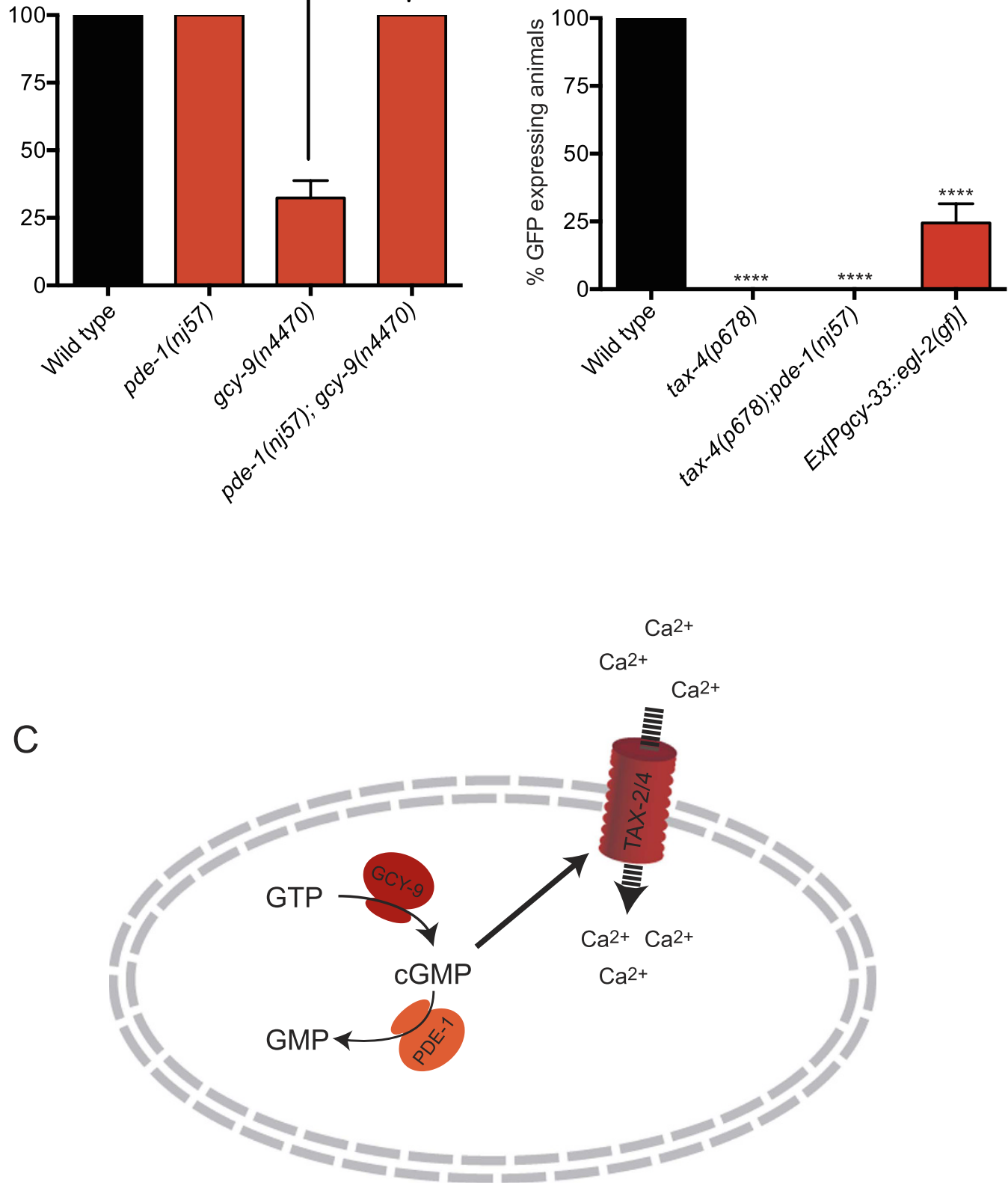

Figure 3. cGMP Levels Regulate $f l p-19:: G F P$ Expression in the BAG Neurons. (A) Quantification of the $f l p-$ 19::GFP reporter suggests that the guanylate cyclase GCY-9 and the phosphodiesterase PDE-1 exhibit opposing regulation of cGMP levels in the BAG neurons. The $p d e-1$ (nj57) mutant exhibits wild type expression of $f l p-$ 19::GFP in the BAG neurons while $g c y-9(n 4470)$ mutants exhibit a strong defect in expression. In $g c y-9(n 4470)$; pde-1(nj57) double mutant animals, flp-19::GFP expression in the BAG neurons is restored, suggesting that cGMP levels in the BAG neurons regulate $f l p-19:: G F P$ expression. See Materials and Methods for neuronal scoring criteria used. $\mathrm{n}>50$. ${ }^{* * *} \mathrm{P}<0.0001$. (B) $f l p-19:: G F P$ in the BAG neurons is completely abrogated in tax-4(p678) mutant animals. Furthermore, the tax-4(p678) mutant defect in $f l p-19:: G F P$ expression cannot be rescued by mutation in $p d e-1$ indicating that tax -4 acts downstream of cGMP signaling. Expression of an constitutively active form of EGL-2 in the BAGs also reduces $f l p-19:: G F P$ expression indicating that $f l p$ 19::GFP expression is activity dependent. See Materials and Methods for neuronal scoring criteria used. $\mathrm{n}>50$. ${ }_{* * * * P}<0.0001$. (C) Schematic model of cGMP regulation in the BAG neurons. GCY-9 converts GTP into cGMP, while PDE- 1 catalyzes the conversion of cGMP into GMP. cGMP opens the TAX-2/TAX-4 channels leading to an influx of calcium and activation of the neuron.

functionally-related neurons. However, we did not observe loss of $f l p$ - $19:: G F P$ expression in the $g c y$ - 35 or $g c y$-36 mutants or the quadruple $g c y$-31 gcy-36; gcy-33; gcy-35 mutant strain (data not shown), suggesting that another pathway of regulation, independent of $\mathrm{O}_{2}$ sensing, regulates the expression of $f l p-19:: G F P$ through the TAX-2/ 


\begin{tabular}{|c|c|c|}
\hline Strain & Defects in BAGs & $\mathbf{n}$ \\
\hline \multicolumn{3}{|l|}{ Neuropeptide } \\
\hline$e g l-3(n r 2090)$ & NO & 62 \\
\hline$u n c-31(e 169)$ & NO & 56 \\
\hline \multicolumn{3}{|l|}{ Neurotransmitter } \\
\hline unc-13(e1091) & NO & 51 \\
\hline eat-4(ky5) & NO & 58 \\
\hline$s n b-1(m d 247)$ & NO & 67 \\
\hline \multicolumn{3}{|c|}{ Activity-related genes } \\
\hline$c r h-1(t z 2)$ & YES & 111 \\
\hline$c r h-1(n 3315)$ & YES & 75 \\
\hline kin-2(ce179) & YES & 193 \\
\hline jun-1(gk557) & $\mathrm{NO}$ & 90 \\
\hline egl-4(n479) & NO & 41 \\
\hline$c m k-1(o k 287)$ & NO & 58 \\
\hline$c k k-1($ ok1033) & NO & 51 \\
\hline rgs-3(ok2288) & NO & 51 \\
\hline
\end{tabular}

Table 1. Genes Tested for Expression of $f l p-19:: G F P$ in the BAG Neurons. We crossed the $f l p-19:: G F P$ reporter with mutants for neuropeptide function, neurotransmission and synaptic regulators and other activityrelated genes. From the screen, we found that $c r h-1$ and $k i n-2$ are necessary for the proper expression of the $f l p-$ 19::GFP reporter.

TAX-4 channels in the URX neurons. To confirm that the BAG neurons are present in tax-4 mutant animals, we crossed tax-4(p678) mutant animals with an independent reporter for BAG neurons (egl-13::GFP), and observed no loss of expression (Figure S1B). Furthermore, we found that loss of $p d e-1$ was not able to restore the tax-4(p678) mutant flp-19::GFP expression, indicating that tax-4 acts downstream of cGMP regulation, and that the channels are necessary for the expression of $f l p-19:: G F P$ regardless of the levels of cGMP (Fig. 3B).

The data we have presented suggest that activity of the BAG neurons is important for the expression of $f l p-$ 19::GFP. To reinforce this assertion, we inactivated the BAG neurons by expressing a constitutively-active EGL2(GF) potassium channel using the $g c y$-33 promoter $^{4,37,38}$. We found that animals carrying the Pgcy-33::egl-2(gf) transgene exhibit a decrease in the expression of $f l p-19:: G F P$ in the BAGs (Fig. 3B).

Taken together, our data show that GCY-9 and the downstream cGMP-regulated TAX-2/TAX-4 channels are required for the expression of $f l p-19:: G F P$ in the BAG neurons. These data suggest that the conversion of GTP to cGMP by GCY- 9 triggers opening of the TAX-2/TAX-4 channels, resulting in calcium influx and that this change in activity regulates the transcription of the neuropeptide reporter $f l p-19:: G F P$ (Fig. 3C).

flp-19::GFP Expression Does not Require Neuropeptide or Neurotransmitter Signaling. We have shown that the expression of $f l p-19:: G F P$ is sensitive to GCY-9 and TAX-4-controlled activity. We next asked whether the expression of $f l p-19:: G F P$ was regulated by neuropeptide and neurotransmitter signaling, through either an autocrine or paracrine fashion. We first tested whether $f l p-19:: G F P$ expression was affected by abolishing neuropeptide signaling. To this end, we crossed the $f l p-19:: G F P$ reporter with two mutants that do not have proper neuropeptide signaling: egl-3(nr2090), responsible for maturation of neuropeptides, and unc-31(e169), involved in dense core vesicle function ${ }^{39-41}$. The expression of $f l p-19:: G F P$ in the BAG neurons in egl-3 and unc-31 mutants was unchanged when compared to wild type (Table 1). The BAG neurons are glutamatergic as they express the vesicular glutamate transporter EAT- $4^{42,43}$. We found that the glutamatergic function of the BAG neurons is not required for the regulation of $f l p-19:: G F P$ as expression is unchanged in an eat-4(ky5) mutant (Table 1). Furthermore, we asked if neurotransmitter release through synaptic vesicle exocytosis was required for $f l p-19:: G F P$ expression. We crossed $f l p-19:: G F P$ with the unc-13(e1091) mutant, defective in neurotransmission due to compromised synaptic vesicle fusion and the $s n b-1(m d 247)$ mutant, defective in synaptic transmis$\operatorname{sion}^{44-46}$. We found that $u n c-13$ and $s n b-1$ mutant animals exhibit wild type expression of $f l p-19:: G F P$ in the BAG neurons (Table 1). These data suggest that the regulation of $f l p-19:: G F P$ in the BAG neurons occurs through a BAG-intrinsic mechanism.

CRH-1/CREB, an Activity-dependent Transcription Factor Acts in Parallel to GCY-9 to Regulate flp-19::GFP Expression in the BAG Neurons. In order to better understand the mechanism through which flp-19::GFP is regulated, we studied mutants of various genes involved in activity-dependent expression in the nervous system (Table 1). Our screen found that both crh-1 and kin-2 mutants display defects in the expression of the $f$ lp-19:: GFP reporter in the BAG neurons. CRH-1 is the C. elegans homolog of CREB and functions in neurons to regulate various behaviors. $\mathrm{CRH}-1$ regulates lifespan, tap habituation and it has been linked with the control of thermotaxis behavior from the AFD neurons ${ }^{47-49}$. KIN-2 is the homolog of the regulatory subunit of cAMP-dependent protein kinase (PKA) and it can regulate CREB activity through a cascade of phosphorylation events $^{50-54}$. We found that two independent null alleles of $c r h-1$ ( $t z 2$ and $\left.n 3315\right)$ exhibit reduced expression of flp-19::GFP in the BAG neurons (Fig. 4A). In addition, kin-2(ce179) mutants show a similar phenotype to $c r h-1$ 
A

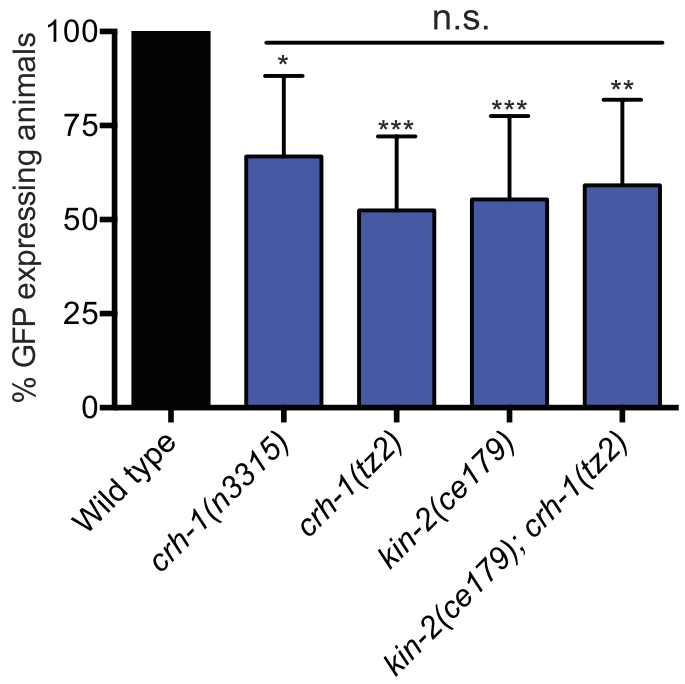

B

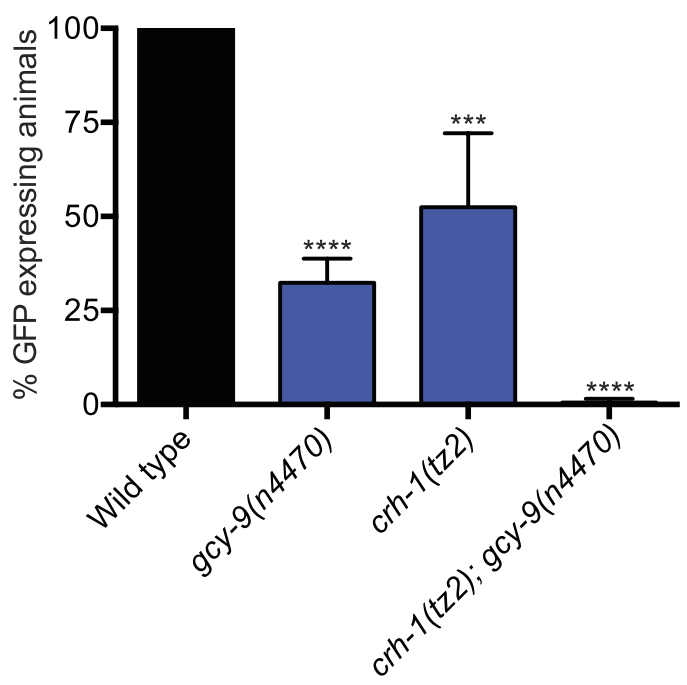

Figure 4. CREB/CRH-1 Acts in Parallel to GCY-9 to Regulate $f l p-19:: G F P$ Expression in the BAG Neurons. (A) Quantification of the defects in $f$ lp-19::GFP expression in the BAG neurons in crh-1 mutant alleles $t z 2$ and $n 3315$ and the kin-2(ce179) mutant. Both crh-1 and kin-2 mutants show a similar penetrance of defects in the expression of $f l p-19:: G F P$ in the BAG neurons. Furthermore, the kin-2(ce179); crh-1(tz2) double mutant defect is not significantly different to either single mutant, suggesting that they act in the same genetic pathway. See Materials and Methods for neuronal scoring criteria used. $\mathrm{n}>50 .{ }^{\star} \mathrm{P}<0.05,{ }^{* \star} \mathrm{P}<0.01,{ }^{* * *} \mathrm{P}<0.0001$, n.s. = not significantly different from wild type. (B) Quantification of the defects in $f l p-19:: G F P$ expression in the BAG neurons in $g c y-9(n 4470), c r h-1(t z 2)$ and the $g c y-9(n 4470)$; $c r h-1(t z 2)$ double mutant. While $c r h-1$ mutants show a partial defect in $f l p-19:: G F P$ expression, in the $c r h-1(t z 2) ; g c y-9(n 4470)$ double mutant $f l p$ 19::GFP expression is undetectable. This indicates that $c r h-1$ acts independently to the $g c y$ - 9 regulatory pathway. See Materials and Methods for neuronal scoring criteria used. $\mathrm{n}>50$. ${ }^{* * *} \mathrm{P}<0.001,{ }^{* * * \star \mathrm{P}}<0.0001$.

mutants, and the crh-1(tz2); kin-2(ce179) double mutant is not significantly different from either single mutant, suggesting that they function in the same genetic pathway (Fig. 4A). We speculated that CRH-1 might be the downstream effector in the GCY-9 cascade. To ask whether $g c y-9$ and $c r h-1$ act in the same genetic pathway to control flp-19::GFP expression we constructed a $g c y-9(n 4470)$; crh-1(tz2) double mutant. Surprisingly, we found that these double mutant animals exhibited complete abrogation of $f l p-19:: G F P$ expression (Fig. 4B). We confirmed that the BAG neurons are present in the $g c y-9(n 4470)$; crh-1(tz2) double mutant by examining a $f l p-$ 13::GFP transgene (Figure S1C). Together, these data suggest that $g c y-9$ and $c r h-1$ act in two separate genetic pathways to regulate $f l p-19::$ GFP expression (Fig. 5). 


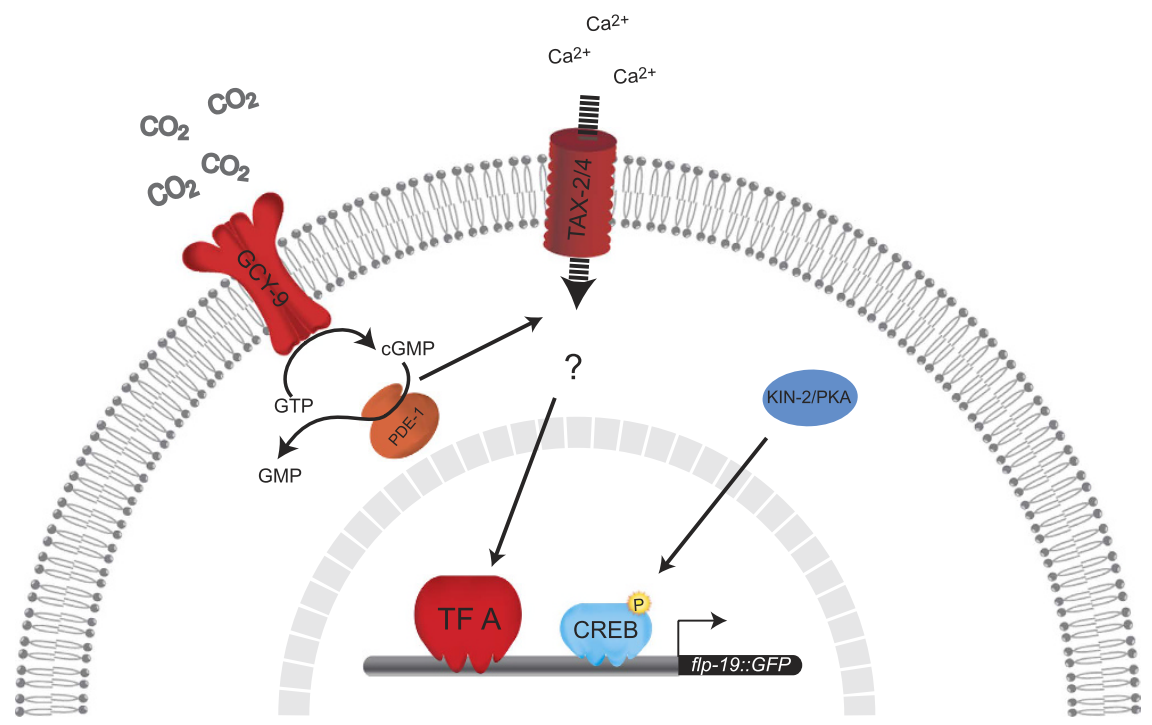

Figure 5. Expression of $f l p-19::$ GFP in the BAG Neurons is Controlled by Parallel Pathways. Schematic representation of the proposed model of regulation of $f l p-19:: G F P$ expression in BAG neurons. The GCY-9 receptor binds $\mathrm{CO}_{2}$ converting GTP into cGMP. cGMP triggers opening of the cyclic nucleotide-gated TAX-2/ TAX-4 cation channels, allowing $\mathrm{Ca}^{2+}$ to enter the neuron, leading to regulation of an unknown transcription factor (TF A) that positively regulates the expression of $f l p-19:: G F P$. PDE-1 reduces the levels of cGMP, opposing the action of GCY-9. In parallel, CRH-1 regulates the expression of $f l p-19:: G F P$ by a signaling pathway involving the cAMP-dependent protein kinase KIN-2.

In summary, we propose a model where $f l p-19:: G F P$ expression is regulated by two parallel pathways: 1) GCY-9 regulation of the TAX-2/TAX-4 cation channels through the control of cGMP levels, signaling to an unknown transcription factor and 2) $\mathrm{CRH}-1 / \mathrm{CREB}$ regulation by the kinase $\mathrm{KIN}-2$. likely through a $\mathrm{CO}_{2}$-independent signaling pathway (Fig. 5).

\section{Discussion}

Activity-dependent regulation is common occurrence in sensory neurons and can contribute in changes in behavior. Chemoreceptor genes can be regulated by different mechanisms such as developmental changes, neuronal activation, or in a paracrine fashion through pheromones. Transcriptional changes of chemoreceptors might be a strategy to modulate external responses. For example, the TAX-2/TAX-4 calcium channel regulates the expression of chemoreceptors such as STR-2 and SRD-1 in the AWC and ASI neurons respectively ${ }^{11,55}$. In addition, TRPV channels control the biosynthesis of serotonin through regulation of tryptophan hydroxylase expression in the ADF neurons ${ }^{56}$.

We have presented data to show that $f l p-19:: G F P$ expression in the BAG neurons is regulated by two parallel pathways. The GCY-9 pathway controls $f l p-19:: G F P$ expression by modulating the levels of cGMP, counterbalanced by the phosphodiesterase PDE-1. PDEs and GCYs are known to function together in other neurons to regulate activity. For example, gcy-12 and pde-2 control cGMP levels to determine body size in C. elegans ${ }^{57}$. Similarly, in the AFD neurons, the opposing roles of GCY-8 and PDE-2, control C. elegans thermotaxis behavior ${ }^{58}$. It might be possible that PDE-1 is involved in sensing the levels of $\mathrm{CO}_{2}$ in the BAG neurons; potentially to set a quantitative threshold or temporal window of TAX-2/TAX-4 channel opening. We have not identified the effector downstream the TAX-2/TAX-4 signaling, but we speculate that it may be a transcription factor regulated by calcium or calmodulin dependent activation. In parallel to GCY-9, CRH-1/CREB also controls the level of flp-19::GFP through the activity of the PKA kinase. As PKA is a cAMP regulated kinase, this may suggest that a cAMP-regulated pathway controls $f l p-19:: G F P$ expression through CRH-1.

It is necessary to further study the implications of the two parallel pathways we have identified to understand the advantages they may provide in the natural habitat of C. elegans. Interestingly worms are attracted to $\mathrm{CO}_{2}$ as dauers while $\mathrm{L} 4$ larvae avoid $\mathrm{CO}_{2}{ }^{5,6,34}$. Furthermore it has been shown how in juvenile infective stages of parasitic worms (related to C. elegans) BAG neurons are involved in the attraction to $\mathrm{CO}_{2}{ }^{59}$. It might be possible that this change in the attraction/repulsion to $\mathrm{CO}_{2}$ is regulated by the expression of neuropeptides such as FLP-19. When worms are grown in low $\mathrm{O}_{2}$ levels, they become attracted to lower levels of $\mathrm{O}_{2}$, instead of being repelled. This change of behavior is due to changes in the expression level of guanylate cyclases that detect $\mathrm{O}_{2}$. It might be possible that similar adaptation occurs when worms are grown in hypercapnic conditions, and the transcriptional regulation of $f l p-19$ could be involved in such adaptation. However, the role of $f l p-19$ may not be directly related to $\mathrm{CO}_{2}$ sensing, but to other broader functions. Indeed, the BAG neurons are involved in lifespan regulation, as is the $\mathrm{CRH}-1$ transcription factor, therefore it would be interesting to examine whether $f l p-19$ mutant animals display defects in longevity.

$f l p-19$ is expressed in a subset of neurons that are distinct in class and function: from oxygen (URX/BAG) and carbon dioxide sensing (BAG), chemotaxis (AWA), egg laying (HSN) or pheromone sensing (CEM). Similar 
means of $f l p-19$ activity-dependent regulation maybe present in these other neurons through different molecular pathways, providing this neuropeptide with multiple layers of control that may be required in particular ephemeral habitats.

\section{Methods}

Strains used in this study. Strains were grown using standard growth conditions on NGM agar at $20^{\circ} \mathrm{C}$ on Escherichia coli OP50 ${ }^{60}$. Transgenic animals were created as previously described ${ }^{61}$. Strain information is detailed in Table S1.

Constructs and generation of transgenic worms. The Pgcy-33::gcy-9 cDNA rescue construct was generated by cloning the $1 \mathrm{~kb} g c y$-33 promoter using HindIII and BamHI and $g c y$-9cDNA, using KpnI and NheI into pPD49.26 vector into multiple cloning sites 1 and 2 respectively (Fire Vector Kit). Transgenic animals were obtained through microinjection ${ }^{61}$. The construct was injected into young adult hermaphrodites as a simple array (20 ng/ul Pgcy-33::gcy-9 cDNA, $10 \mathrm{ng} / \mathrm{ul}$ myo-3::RFP as co-injection marker).

The $B A G:: e g l-2(g f)$ construct was generated by cloning the $1 \mathrm{~kb} g c y$-33 promoter using HindIII and BamHI and egl-2(gf)cDNA (kind gift of Mario De Bono) using KpnI into the pPD49.26 vector into multiple cloning sites

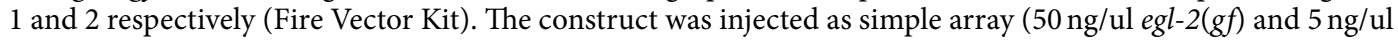
myo-3::RFP as co-injection marker).

Microscopy. Worms were anesthetized in $20 \mathrm{mM} \mathrm{NaN}_{3}$ on $5 \%$ agarose on glass slides and images were taken using an upright fluorescence microscope (Zeiss, AXIO Imager M2) and ZEN software (version 2.0). Neuronal scoring: Neurons were given a numerical value according to their expression levels. Wild-type expression scored 1, decreased expression scored 0.5 and abolished expression scored 0. Percentage of GFP expressing animals was then correlated to the theoretical maximum score using the equation below.

$$
\% \text { of GFP expressing animals }=\frac{\text { observed score }\left(n 1^{*} 1\right)+(n 2 * 0.5)+n 3^{*} 0}{\text { theoretical score }(n 1+n 2+n 3)^{*} 1} \times 100
$$

Statistical analysis. Statistical analysis was performed in GraphPad Prism 6 using one-way ANOVA with Newman-Keuls Multiple Comparison Test. Values are expressed as mean $+/-$ s.d. Differences with a $\mathrm{P}$ value $<0.05$ were considered significant.

\section{References}

1. Hedgecock, E. M. \& Russell, R. L. Normal and mutant thermotaxis in the nematode Caenorhabditis elegans. Proc Natl Acad Sci USA 72, 4061-4065 (1975)

2. Mori, I. \& Ohshima, Y. Neural regulation of thermotaxis in Caenorhabditis elegans. Nature 376, 344-348 (1995).

3. Gray, J. M. et al. Oxygen sensation and social feeding mediated by a C. elegans guanylate cyclase homologue. Nature 430, 317-322 (2004).

4. Cheung, B. H., Cohen, M., Rogers, C., Albayram, O. \& de Bono, M. Experience-dependent modulation of C. elegans behavior by ambient oxygen. Curr Biol 15, 905-917 (2005).

5. Bretscher, A. J., Busch, K. E. \& de Bono, M. A carbon dioxide avoidance behavior is integrated with responses to ambient oxygen and food in Caenorhabditis elegans. Proc Natl Acad Sci USA 105, 8044-8049, doi: 10.1073/pnas.0707607105 (2008).

6. Hallem, E. A. \& Sternberg, P. W. Acute carbon dioxide avoidance in Caenorhabditis elegans. Proc Natl Acad Sci USA 105, 8038-8043, doi: 10.1073/pnas.0707469105 (2008).

7. Ward, S. Chemotaxis by the nematode Caenorhabditis elegans: identification of attractants and analysis of the response by use of mutants. Proc Natl Acad Sci USA 70, 817-821 (1973).

8. Croll, N. A. Components and patterns in the behavior of the nematode Caenorhabditis elegans. J. Zool. 176, 159-176 (1975).

9. Lakhina, V. et al. Genome-wide functional analysis of CREB/long-term memory-dependent transcription reveals distinct basal and memory gene expression programs. Neuron 85, 330-345, doi: 10.1016/j.neuron.2014.12.029 (2015).

10. Uhl, G. R. \& Nishimori, T. Neuropeptide gene expression and neural activity: assessing a working hypothesis in nucleus caudalis and dorsal horn neurons expressing preproenkephalin and preprodynorphin. Cell Mol Neurobiol 10, 73-98 (1990).

11. Peckol, E. L., Troemel, E. R. \& Bargmann, C. I. Sensory experience and sensory activity regulate chemosensory receptor gene expression in Caenorhabditis elegans. Proc Natl Acad Sci USA 98, 11032-11038. (2001).

12. Gruner, M. et al. Feeding state, insulin and NPR-1 modulate chemoreceptor gene expression via integration of sensory and circuit inputs. PLoS Genet 10, e1004707, doi: 10.1371/journal.pgen.1004707 (2014).

13. Laurent, P. et al. Decoding a neural circuit controlling global animal state in C. elegans. Elife 4, doi: 10.7554/eLife.04241 (2015).

14. Dillon, J., Holden-Dye, L., O'Connor, V. \& Hopper, N. A. Context-dependent regulation of feeding behaviour by the insulin receptor, DAF-2, in Caenorhabditis elegans. Invert Neurosci 16, 4, doi: 10.1007/s10158-016-0187-2 (2016).

15. Li, C., Nelson, L. S., Kim, K., Nathoo, A. \& Hart, A. C. Neuropeptide gene families in the nematode Caenorhabditis elegans. Ann N Y Acad Sci 897, 239-252 (1999).

16. Turek, M., Besseling, J., Spies, J. P., Konig, S. \& Bringmann, H. Sleep-active neuron specification and sleep induction require FLP-11 neuropeptides to systemically induce sleep. Elife 5, doi: 10.7554/eLife.12499 (2016).

17. Nelson, M. D. et al. FMRFamide-like FLP-13 neuropeptides promote quiescence following heat stress in Caenorhabditis elegans. Curr Biol 24, 2406-2410, doi: 10.1016/j.cub.2014.08.037 (2014).

18. Ringstad, N. \& Horvitz, H. R. FMRFamide neuropeptides and acetylcholine synergistically inhibit egg-laying by C. elegans. Nat Neurosci 11, 1168-1176, doi: 10.1038/nn.2186 (2008).

19. Rogers, C. et al. Inhibition of Caenorhabditis elegans social feeding by FMRFamide-related peptide activation of NPR-1. Nat Neurosci 6, 1178-1185, doi: 10.1038/nn1140nn1140 (2003).

20. Sharabi, K. et al. The response to high CO2 levels requires the neuropeptide secretion component HID-1 to promote pumping inhibition. PLoS Genet 10, e1004529, doi: 10.1371/journal.pgen.1004529 (2014)

21. Rogers, C., Persson, A., Cheung, B. \& de Bono, M. Behavioral motifs and neural pathways coordinating O2 responses and aggregation in C. elegans. Curr Biol 16, 649-659, doi: S0960-9822(06)01316-910.1016/j.cub.2006.03.023 (2006).

22. Zimmer, M. et al. Neurons detect increases and decreases in oxygen levels using distinct guanylate cyclases. Neuron 61, 865-879, doi: S0896-6273(09)00158-510.1016/j.neuron.2009.02.013 (2009). 
23. Carrillo, M. A., Guillermin, M. L., Rengarajan, S., Okubo, R. P. \& Hallem, E. A. O2-sensing neurons control CO2 response in C. elegans. J Neurosci 33, 9675-9683, doi: 10.1523/JNEUROSCI.4541-12.2013 (2013).

24. Liu, T. \& Cai, D. Counterbalance between BAG and URX neurons via guanylate cyclases controls lifespan homeostasis in C. elegans. EMBO J 32, 1529-1542, doi: 10.1038/emboj.2013.75 (2013).

25. Kim, K. \& Li, C. Expression and regulation of an FMRFamide-related neuropeptide gene family in Caenorhabditis elegans. J Comp Neurol 475, 540-550 (2004).

26. Brandt, J. P. et al. A single gene target of an ETS-family transcription factor determines neuronal CO2-chemosensitivity. PLoS One 7, e34014, doi: 10.1371/journal.pone.0034014 (2012).

27. Gramstrup Petersen, J. et al. EGL-13/SoxD specifies distinct O2 and CO2 sensory neuron fates in Caenorhabditis elegans. PLoS Genet 9, e1003511, doi: 10.1371/journal.pgen.1003511 (2013).

28. Rojo Romanos, T., Petersen, J. G., Riveiro, A. R. \& Pocock, R. A novel role for the zinc-finger transcription factor EGL-46 in the differentiation of gas-sensing neurons in Caenorhabditis elegans. Genetics 199, 157-163, doi: 10.1534/genetics.114.172049 (2015).

29. Perkins, L. A., Hedgecock, E. M., Thomson, J. N. \& Culotti, J. G. Mutant sensory cilia in the nematode Caenorhabditis elegans. Dev Biol 117, 456-487 (1986).

30. Wicks, S. R., de Vries, C. J., van Luenen, H. G. \& Plasterk, R. H. CHE-3, a cytosolic dynein heavy chain, is required for sensory cilia structure and function in Caenorhabditis elegans. Dev Biol 221, 295-307 (2000).

31. Brear, A. G., Yoon, J., Wojtyniak, M. \& Sengupta, P. Diverse cell type-specific mechanisms localize G protein-coupled receptors to Caenorhabditis elegans sensory cilia. Genetics 197, 667-684, doi: 10.1534/genetics.114.161349 (2014).

32. Mukhopadhyay, A., Deplancke, B., Walhout, A. J. \& Tissenbaum, H. A. C. elegans tubby regulates life span and fat storage by two independent mechanisms. Cell Metab 2, 35-42, doi: 10.1016/j.cmet.2005.06.004 (2005).

33. Ikeda, A. et al. Microtubule-associated protein 1A is a modifier of tubby hearing (moth1). Nat Genet 30, 401-405, doi: 10.1038/ ng838 (2002)

34. Hallem, E. A. et al. Receptor-type guanylate cyclase is required for carbon dioxide sensation by Caenorhabditis elegans. Proc Natl Acad Sci USA 108, 254-259, doi: 10.1073/pnas.1017354108 (2011).

35. Smith, E. S., Martinez-Velazquez, L. \& Ringstad, N. A chemoreceptor that detects molecular carbon dioxide. J Biol Chem 288, 37071-37081, doi: 10.1074/jbc.M113.517367 (2013).

36. Chang, A. J., Chronis, N., Karow, D. S., Marletta, M. A. \& Bargmann, C. I. A distributed chemosensory circuit for oxygen preference in C. elegans. PLoS Biol 4, e274, doi: 06-PLBI-RA-0332R310.1371/journal.pbio.0040274 (2006).

37. Coates, J. C. \& de Bono, M. Antagonistic pathways in neurons exposed to body fluid regulate social feeding in Caenorhabditis elegans. Nature 419, 925-929, doi: 10.1038/nature01170nature01170 (2002).

38. Weinshenker, D., Wei, A., Salkoff, L. \& Thomas, J. H. Block of an ether-a-go-go-like $\mathrm{K}(+)$ channel by imipramine rescues egl-2 excitation defects in Caenorhabditis elegans. J Neurosci 19, 9831-9840 (1999).

39. Avery, L., Bargmann, C. I. \& Horvitz, H. R. The Caenorhabditis elegans unc-31 gene affects multiple nervous system-controlled functions. Genetics 134, 455-464 (1993).

40. Speese, S. et al. UNC-31 (CAPS) is required for dense-core vesicle but not synaptic vesicle exocytosis in Caenorhabditis elegans. J Neurosci 27, 6150-6162, doi: 27/23/615010.1523/JNEUROSCI.1466-07.2007 (2007).

41. Kass, J., Jacob, T. C., Kim, P. \& Kaplan, J. M. The EGL-3 proprotein convertase regulates mechanosensory responses of Caenorhabditis elegans. J Neurosci 21, 9265-9272 (2001).

42. Serrano-Saiz, E. et al. Modular control of glutamatergic neuronal identity in C. elegans by distinct homeodomain proteins. Cell 155, 659-673, doi: 10.1016/j.cell.2013.09.052 (2013).

43. Lee, R. Y., Sawin, E. R., Chalfie, M., Horvitz, H. R. \& Avery, L. EAT-4, a homolog of a mammalian sodium-dependent inorganic phosphate cotransporter, is necessary for glutamatergic neurotransmission in caenorhabditis elegans. J Neurosci 19, 159-167 (1999).

44. Nonet, M. L., Saifee, O., Zhao, H., Rand, J. B. \& Wei, L. Synaptic transmission deficits in Caenorhabditis elegans synaptobrevin mutants. J Neurosci 18, 70-80 (1998).

45. Richmond, J. E., Davis, W. S. \& Jorgensen, E. M. UNC-13 is required for synaptic vesicle fusion in C. elegans. Nat Neurosci 2, 959-964 (1999).

46. Miller, K. G. et al. A genetic selection for Caenorhabditis elegans synaptic transmission mutants. Proc Natl Acad Sci USA 93, 12593-12598 (1996).

47. Mair, W. et al. Lifespan extension induced by AMPK and calcineurin is mediated by CRTC-1 and CREB. Nature 470, 404-408, doi: 10.1038/nature09706 (2011).

48. Timbers, T. A. \& Rankin, C. H. Tap withdrawal circuit interneurons require CREB for long-term habituation in Caenorhabditis elegans. Behav Neurosci 125, 560-566, doi: $10.1037 /$ a0024370 (2011).

49. Nishida, Y., Sugi, T., Nonomura, M. \& Mori, I. Identification of the AFD neuron as the site of action of the CREB protein in Caenorhabditis elegans thermotaxis. EMBO Rep 12, 855-862, doi: 10.1038/embor.2011.120 (2011).

50. Gonzalez, G. A. \& Montminy, M. R. Cyclic AMP stimulates somatostatin gene transcription by phosphorylation of CREB at serine 133. Cell 59, 675-680 (1989).

51. Brindle, P., Linke, S. \& Montminy, M. Protein-kinase-A-dependent activator in transcription factor CREB reveals new role for CREM repressors. Nature 364, 821-824, doi: 10.1038/364821a0 (1993).

52. Quinn, P. G. Distinct activation domains within cAMP response element-binding protein (CREB) mediate basal and cAMPstimulated transcription. J Biol Chem 268, 16999-17009 (1993).

53. Mayr, B. \& Montminy, M. Transcriptional regulation by the phosphorylation-dependent factor CREB. Nat Rev Mol Cell Biol 2, 599-609, doi: 10.1038/35085068 (2001).

54. Pastok, M. W. et al. Structural diversity of the cAMP-dependent protein kinase regulatory subunit in Caenorhabditis elegans. Cell Signal 25, 168-177, doi: 10.1016/j.cellsig.2012.09.006 (2013).

55. Troemel, E. R., Sagasti, A. \& Bargmann, C. I. Lateral signaling mediated by axon contact and calcium entry regulates asymmetric odorant receptor expression in C. elegans. Cell 99, 387-398 (1999).

56. Zhang, S., Sokolchik, I., Blanco, G. \& Sze, J. Y. Caenorhabditis elegans TRPV ion channel regulates 5HT biosynthesis in chemosensory neurons. Development 131, 1629-1638 (2004).

57. Fujiwara, M. et al. The Importance of cGMP Signaling in Sensory Cilia for Body Size Regulation in Caenorhabditis elegans. Genetics 201, 1497-1510, doi: 10.1534/genetics.115.177543 (2015).

58. Wang, D., O'Halloran, D. \& Goodman, M. B. GCY-8, PDE-2, and NCS-1 are critical elements of the cGMP-dependent thermotransduction cascade in the AFD neurons responsible for C. elegans thermotaxis. J Gen Physiol 142, 437-449, doi: 10.1085/ jgp.201310959 (2013).

59. Hallem, E. A. et al. A sensory code for host seeking in parasitic nematodes. Curr Biol 21, 377-383, doi: 10.1016/j.cub.2011.01.048 (2011)

60. Brenner, S. The genetics of Caenorhabditis elegans. Genetics 77, 71-94 (1974).

61. Mello, C. C., Kramer, J. M., Stinchcomb, D. \& Ambros, V. Efficient gene transfer in C.elegans: extrachromosomal maintenance and integration of transforming sequences. Embo J 10, 3959-3970 (1991). 


\section{Acknowledgements}

We thank members of Pocock laboratory for comments on the manuscript. Some strains used in this study were provided by the Caenorhabditis Genetics Center, which is funded by NIH Office of Research Infrastructure Programs (P40 OD010440) and by Shohei Mitani at the National Bioresource Project (Japan). We also thank Mario De Bono for the gift of egl-2(gf) cDNA. This work was supported by a grant from the Lundbeck Foundation (R140-2013-13330) and veski innovation fellowship (VIF\#23) to RP.

\section{Author Contributions}

T.R.R. and J.G.P. performed the experiments, T.R.R. and R.P. wrote the manuscript.

\section{Additional Information}

Supplementary information accompanies this paper at http://www.nature.com/srep

Competing financial interests: The authors declare no competing financial interests.

How to cite this article: Romanos, T. R. et al. Control of Neuropeptide Expression by Parallel Activitydependent Pathways in Caenorhabditis elegans. Sci. Rep. 7, 38734; doi: 10.1038/srep38734 (2017).

Publisher's note: Springer Nature remains neutral with regard to jurisdictional claims in published maps and institutional affiliations.

(c) (i) This work is licensed under a Creative Commons Attribution 4.0 International License. The images or other third party material in this article are included in the article's Creative Commons license, unless indicated otherwise in the credit line; if the material is not included under the Creative Commons license, users will need to obtain permission from the license holder to reproduce the material. To view a copy of this license, visit http://creativecommons.org/licenses/by/4.0/

(C) The Author(s) 2017 